\begin{tabular}{|c|l|}
\hline Title & Diffusive behavior of a thin particle layer in fluid by hydrodynamic interaction \\
\hline Author(s) & Harada, Shusaku; Otomo, Ryoko \\
\hline Citation & $\begin{array}{l}\text { Physical Review E, 80(6), 066311 } \\
\text { https://doi.org/_0.1103/PhysRevE.80.066311 }\end{array}$ \\
\hline Issue Date & 2009-12 \\
\hline Doc URL & http://hdl.handle.net/2115/42573 \\
\hline Rights & ○2009 The A merican Physical Society \\
\hline Type & article \\
\hline File Information & PRE80_6_066311.pdf \\
\hline
\end{tabular}

Instructions for use 


\title{
Diffusive behavior of a thin particle layer in fluid by hydrodynamic interaction
}

\author{
Shusaku Harada* and Ryoko Otomo \\ Division of Field Engineering for Environment, Graduate School of Engineering, \\ Hokkaido University, N13-W8, Sapporo 060-8628, Japan
}

(Received 15 July 2009; revised manuscript received 21 October 2009; published 15 December 2009)

\begin{abstract}
The hydrodynamic effect on a thin particle layer, which moves relative to fluid by an external force, is investigated theoretically and numerically. Because of the presence of layer ends, the arrangement of particles in the layer is anisotropic and the drag force acting on them varies according to the position. The resulting relative motion of particles brings about the spreading of the layer. We have studied such a diffusive behavior of particle layers, which have various internal arrangements. We have assumed a non-Brownian system in which the particles move relatively owing to only the variance of hydrodynamic force. The hydrodynamic force on each particle was calculated by Stokesian dynamics approach. The results show that the relative motion of particles is greatly influenced by the internal arrangement of the particle layer. In consequence, the overall diffusive motion of particle layer varies with the arrangement even if the particle concentration is similar. It is in contrast to the gradient diffusion of Brownian particles.
\end{abstract}

DOI: 10.1103/PhysRevE.80.066311

PACS number(s): 47.61.Jd

\section{INTRODUCTION}

The relative motion of solid particles to surrounding fluid has been important since early times in various engineering processes such as mixing, separation, and dispersion of particulate materials in fluid. Besides, two- or three-dimensional crystalline arrays of colloidal particles have been made for new optical and electronic devices in recent years, and the motion of particles relative to fluid often plays a significant role in the fabrication of highly ordered crystalline arrays [1].

It is known that the motion of particles in fluid by an external force, such as gravity force, is influenced by the hydrodynamic interaction with their surrounding particles. The hydrodynamic interaction is the momentum interchange of particles due to the disturbed fluid flow by each particle. For example, the mean settling velocity of particles in suspension depends on the particle concentration and it decreases with the increase in the concentration owing to their hydrodynamic interactions [2].

Not only does the hydrodynamic interaction affect the whole motion of suspension but also it causes the relative motion between suspended particles. The resultant fluctuating motion brings about various hydrodynamic effects such as the clustering and the diffusion of particles [3]. In general, the hydrodynamic interactions between particles are longranged under conditions of low particle Reynolds number, since the disturbed flow by the motion of individual particles decays inversely proportional to the distance from the particle. Consequently, these effects are more influential with the motion of finer particles in more viscous fluid.

The diffusion due to the hydrodynamic interaction of particle has been studied by many researchers and it has been called hydrodynamic diffusion or hydrodynamic dispersion [3-9]. In the system which the diffusion lengthscale is much greater than the size of suspended particles, which is often observed in the sedimentation process of fine particle, the

\footnotetext{
*harada@eng.hokudai.ac.jp
}

diffusive property of suspended particles has been treated analogous to that of molecular diffusion. Concerning this system, the numerical simulation and the direct measurement of the fluctuating motion of particles in fluid have suggested that the diffusion coefficient varies with the concentration and it has a strong dependency on the orientation.

On the other hand, in the coating process of particle or the fabrication of two-dimensional particle arrays, the lengthscale of hydrodynamic diffusion is the same order of particle size. In such anisotropic systems, it is difficult to consider the diffusion properties from macroscopic viewpoint like molecular diffusion because the local averaged variables, such as the concentration and the fluctuation velocity, cannot be defined properly. Therefore, it has to be considered as individual motions of constituent particles. The analyses on the diffusion property by taking such an approach have been rarely seen in previous researches.

The purpose of this study is to investigate the hydrodynamic effect on particles in the anisotropic system. As shown in Fig. 1, we consider a thin particle layer, which moves relative to a stationary fluid by an external force. We have assumed the particle in the layer is small and moves slowly, i.e., both fluid and particle inertias can be neglected. In such a system, the hydrodynamic interaction between particles is determined only by the relative position of particles [10] Therefore, the drag force exerted on particles near the layer end is different from that on inside particles due to anisotropic arrangements of their surrounding particles. The dif-

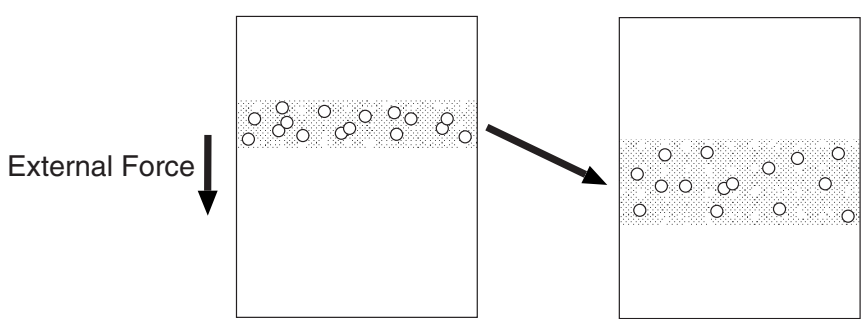

FIG. 1. Schematic diagram of relative motion of a particle layer to stationary fluid by an external force. 
ference of drag force brings about the relative motion of particles in the layer and, consequently, the thickness of the layer may change. From macroscopic viewpoint, it can be interpreted as the diffusion caused by the end effect of hydrodynamic interaction.

We have particularly focused on the relative motion of particles and have investigated such a diffusive process at the level of individual particle theoretically and numerically. The outlines of this paper are as follows. In Sec. II, we begin with an explanation about the calculation method. In Sec. III, the following are discussed: (A) drag force exerted on each of spatially fixed particles consisting of the layer in a fluid; (B) dynamics of regularly arranged particle layer; and (C) dynamics of randomly arranged particle layer. In Sec. III A, we exhibit our results with those by previous studies and show interesting features concerning the dependency of the drag force on particle arrangement inside the layer. In Secs. III B and III C, we show the remarkable changes in the diffusive behavior of particle layer according to the internal arrangement and discuss how the presence of layer ends affects the diffusive properties of particles. In Sec. IV, we conclude our study.

\section{ASSUMPTION AND FORMULATION}

The motion of fine particles which form a thin layer in a stationary fluid is considered. The constant external force is applied to each particle. On the assumption that the particle is adequately small and it moves slowly, the inertias of both particle and fluid are neglected. We consider a non-Brownian system in which the particles move relatively only due to the difference in the hydrodynamic force acting on them.

Figure 1 shows a schematic diagram of the relative motion of particle layer to fluid. The thickness of the particle layer is finite in the direction of external force, while it is infinite in the other directions. The motion of each particle is calculated by Stokesian dynamics approach. The mobility matrix of particles is based on the Rotne-Prager tensor [11], which is based on the multipole expansion of Oseen tensor and Faxén's law [10]. The external force acting on each particle is related to the particle velocity by the mobility matrix as follows:

$$
6 \pi \mu a U_{i}^{\alpha}=F_{i}^{\alpha}+\sum_{\substack{\beta=1 \\(\beta \neq \alpha)}}^{N} M_{i j}\left(x^{\alpha}-x^{\beta}\right) F_{j}^{\beta},
$$

where $a$ is the particle radius, $\mu$ is the viscosity, $\boldsymbol{x}^{\alpha, \beta}, \boldsymbol{U}^{\alpha, \beta}$, and $\boldsymbol{F}^{\alpha, \beta}$ are the position, the velocity, and the external force of particles $\alpha, \beta$, respectively. The mobility matrix $M_{i j}(\boldsymbol{r})$ is given as follows:

$$
M_{i j}(\boldsymbol{r})=\left(\frac{3}{4} a+\frac{1}{4} a^{3} \nabla^{2}\right)\left(\nabla^{2} \delta_{i j}-\frac{\partial^{2}}{\partial r_{i} \partial r_{j}}\right) r
$$

where $r=|\boldsymbol{r}|$.

In this study, the periodic boundary is applied in two directions $(x$ and $y$ ). Ewald summation technique is applied to the mobility matrix in these directions in order to calculate properly the hydrodynamic effect of the further particles. If we set the length of the calculation domain (the lattice width of Ewald sum) in $x$ and $y$ directions to be $L_{1}$ and $L_{2}$, respectively, the lattice vector is defined by $\boldsymbol{r}_{\gamma}=\left(n_{1} L_{1}, n_{2} L_{2}, 0\right)$, where $n_{1}, n_{2}=0, \pm 1, \pm 2, \ldots$. The relationship between the external force and the particle velocity is given as follows $[12,13]$ :

$$
\begin{aligned}
6 \pi \mu a U_{i}^{\alpha}= & F_{i}^{\alpha}+\sum_{n_{1}, n_{2}} \sum_{\substack{\beta=1 \\
(\beta \neq \alpha)}}^{N} M_{i j}^{(1)}\left(\boldsymbol{x}^{\alpha}-\boldsymbol{x}^{\beta}+\boldsymbol{r}_{\gamma}\right) F_{j}^{\beta}+\frac{1}{L_{1} L_{2}} \\
& \times \sum_{\substack{m_{1}, m_{2} \\
(\boldsymbol{k} \neq 0)}}^{N} \sum_{\beta=1}^{N} \exp \left\{-i\left[k_{1}\left(x^{\alpha}-x^{\beta}\right)+k_{2}\left(y^{\alpha}-y^{\beta}\right)\right]\right\} \\
& \times \tilde{M}_{i j}^{(2)}\left(k_{1}, k_{2} ; z^{\alpha}-z^{\beta}\right) F_{j}^{\beta}-M_{i j}^{(2)}(\boldsymbol{r}=0) F_{j}^{\alpha},
\end{aligned}
$$

where $k_{1}=2 \pi m_{1} / L_{1} \quad$ and $\quad k_{2}=2 \pi m_{2} / L_{2} \quad\left(m_{1}, m_{2}\right.$ : $0, \pm 1, \pm 2, \ldots)$. The mobility matrices in real and reciprocal spaces $M_{i j}^{(1)}(\boldsymbol{r}), M_{i j}^{(2)}(\boldsymbol{r})$, and $\tilde{M}_{i j}^{(2)}\left(k_{1}, k_{2} ; z\right)$ are given as follows:

$$
\begin{gathered}
M_{i j}^{(1)}(\boldsymbol{r})=\left(\frac{3}{4} a+\frac{1}{4} a^{3} \nabla^{2}\right)\left(\nabla^{2} \delta_{i j}-\frac{\partial^{2}}{\partial r_{i} \partial r_{j}}\right)[r \operatorname{erfc}(\xi r)], \\
M_{i j}^{(2)}(\boldsymbol{r})=\left(\frac{3}{4} a+\frac{1}{4} a^{3} \nabla^{2}\right)\left(\nabla^{2} \delta_{i j}-\frac{\partial^{2}}{\partial r_{i} \partial r_{j}}\right)[r \operatorname{erf}(\xi r)], \\
\tilde{M}_{i j}^{(2)}\left(k_{1}, k_{2} ; z\right)=\int_{-\infty}^{\infty} \int_{-\infty}^{\infty} M_{i j}^{(2)}(\boldsymbol{r}) e^{i\left(k_{1} x+k_{2} y\right)} d x d y,
\end{gathered}
$$

where $\xi$ is the parameter for lattice sum. The lattice sum of real space shown as the second term of the right-hand side in Eq. (3) is solved by the same procedure as Beenakker [12]. The lattice sum of reciprocal space shown as the third term is solved in the same way as Ichiki [13]. The numerical integration scheme is used for the calculation of a few terms in the above mobility matrices.

In addition, the correction for the short-range interaction is applied to the mobility matrix in the same way as Durlofsky et al. [14]. Rewriting Eq. (3), we obtain the vector form of mobility equation for all particles as follows:

$$
\boldsymbol{U}=\boldsymbol{M}_{G} \cdot \boldsymbol{F},
$$

where $\boldsymbol{U}$ and $\boldsymbol{F}$ indicate the velocity and the force acting on all particles, respectively. Instead of the grand mobility matrix $\boldsymbol{M}_{G}$ in Eq. (7), the following matrix $\overline{\boldsymbol{M}}_{G}$ is used:

$$
\overline{\boldsymbol{M}}_{G}=\left[\boldsymbol{M}_{G}^{-1}+\boldsymbol{R}_{2 B}-\left(\boldsymbol{M}_{2 B}\right)^{-1}\right]^{-1},
$$

where $\boldsymbol{R}_{2 B}$ is the resistance matrix of pair particles and $\boldsymbol{M}_{2 B}$ is the pair mobility matrix given by Eq. (2). The resistance matrix $\boldsymbol{R}_{2 B}$ was solved exactly by Jeffrey and Onishi [15] and their solution is used here. The instantaneous position of particles is calculated by numerical integration of Eq. (7), replacing $\boldsymbol{M}_{G}$ to $\overline{\boldsymbol{M}}_{G}$. 

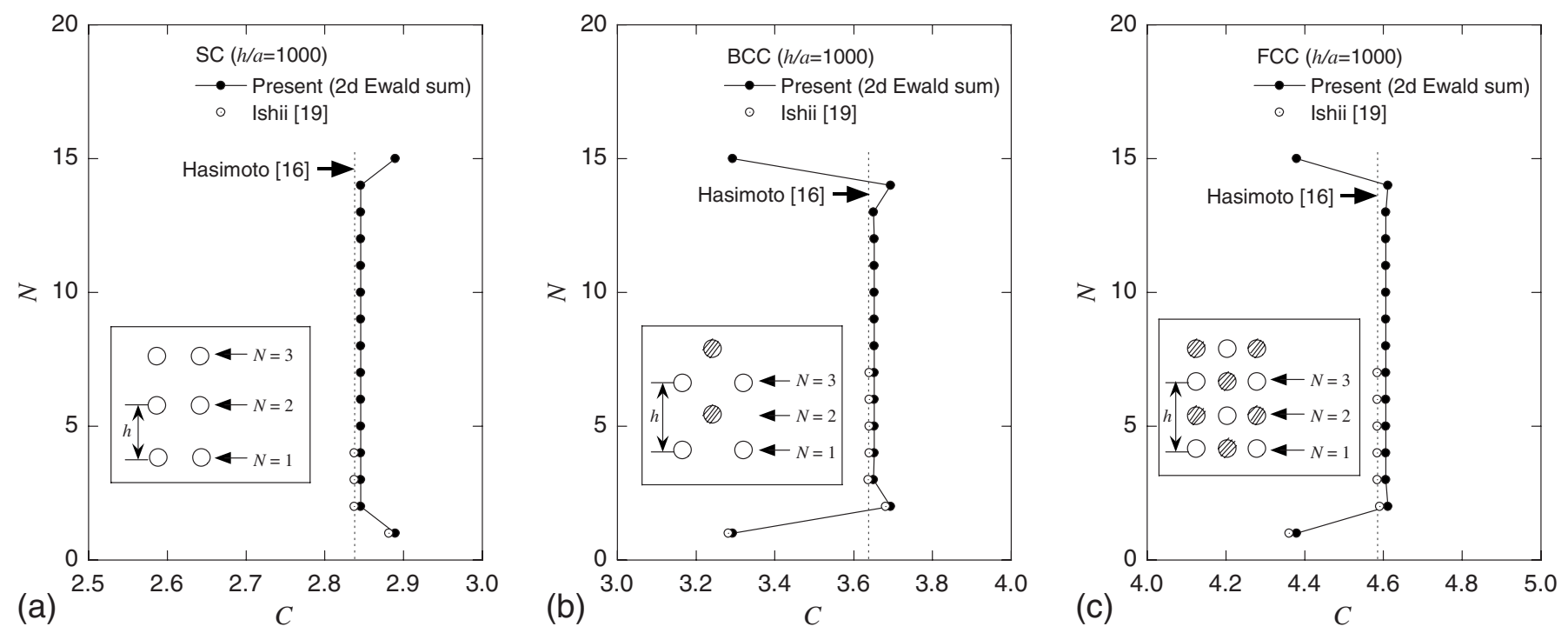

FIG. 2. Drag force acting on a dilute particle layer for (a) SC array, (b) BCC array, and (c) FCC array [C indicates the coefficient in Eq. (9)].

\section{RESULTS AND DISCUSSION}

\section{A. Drag force exerted on regular particle layer}

We have investigated the diffusive behavior caused by the hydrodynamic interaction between particles consisting of a thin layer. We have considered the spreading of the layer resulting from the variance of the drag force on particles in it. It might be expected that the drag exerted on each particle in the layer depends on their relative position, i.e., the drag exerted on particles at the end of the layer differs from that in the center. For the fundamental understanding of these effects, we begin with a discussion about the drag force exerted on particles in a spatially fixed layer. We examined three kinds of arrays with regular arrangement, namely, simple cubic (SC), body-centered cubic (BCC), and facecentered cubic (FCC). The drag coefficients of these arrays in an infinite system (the particle exists endlessly in space) [16-18] and in a semi-infinite system (the end of particle layer exists only in one direction) $[19,20]$ have already been reported.

We first validated our analysis method by comparing it to other previous studies with regard to the drag force exerted on dilute particle arrays. Figure 2 shows the results of the drag coefficient calculation in fixed regular particle layers with finite thickness. In the figure, $N$ indicates the particle number in the layer, and $C$ is the modified coefficient of the Stokes drag, which is defined by following equation:

$$
F=6 \pi \mu a U(1+C a / h)
$$

where $F$ is the drag force, $U$ is the relative velocity of fluid to particle, and $h / a$ is the ratio of unit length of particle separation to particle radius. The definitions of $h$ are shown in the inset of each figure. For all conditions, we set $h / a=1000$, which corresponds to extremely dilute concentration. The total number of particle arrays $N_{L}$ was set to be $15 . N=1$ and $N=N_{L}$ indicate the particles existing on both ends of the layer. Figure 2 also shows the results for the infinite system by Hasimoto [16] and those for the semi-infinite system by Ishii [19], which are $O(a / h)$ solution.

As can be seen in Fig. 2, the profile of drag on particles in the layer is symmetrical because of the linearity of the Stokes equation. The drag coefficients of the central particles in the layer agree well with that of the infinite system by Hasimoto. On the other hand, the drag on particles near the layer end is different from that on the central particles. They agree quantitatively with those in the semi-infinite system by Ishii.

The profile of the drag coefficient near the layer end varies with the internal arrangement. For SC array, the drag coefficient at the end is larger than that in the center, while it is smaller for BCC and FCC. Such interesting features of drag force have already been reported by Ishii. These results indicate that the drag on particles near the end is influenced by the internal arrangement of the layer. In consequence, if these particles were not fixed, their relative motion would vary according to the internal arrangement.

The difference of the drag force at the end of layer is also found in dense system. Figure 3 shows the drag coefficient $K=F / 6 \pi \mu a U$ of the fixed regular particle layers on dense conditions. We set the number of arrays $N_{L}=11$ for all arrangements. The figure also indicates the drag coefficient near the end of semi-infinite particle array by Sangani and Behl [20]. The drag coefficient shows similar profiles to the dilute conditions, i.e., the drag on the end of the layer is larger than that on the central particles for SC, while it is smaller for BCC and FCC. It is also found from Fig. 3 that the difference of the drag decays within the close proximity to the end of the layer even in dense system as well as in dilute system.

Here we show the results only for the concentration $\phi$ $<0.2$ since we considered only the low-order moments of multipole expansion. In consequence, our analysis could not be applied to the denser system. Sangani et al. also calculated the drag on semi-infinite arrays for dense packing limit. According to their results, the difference of the drag near the 

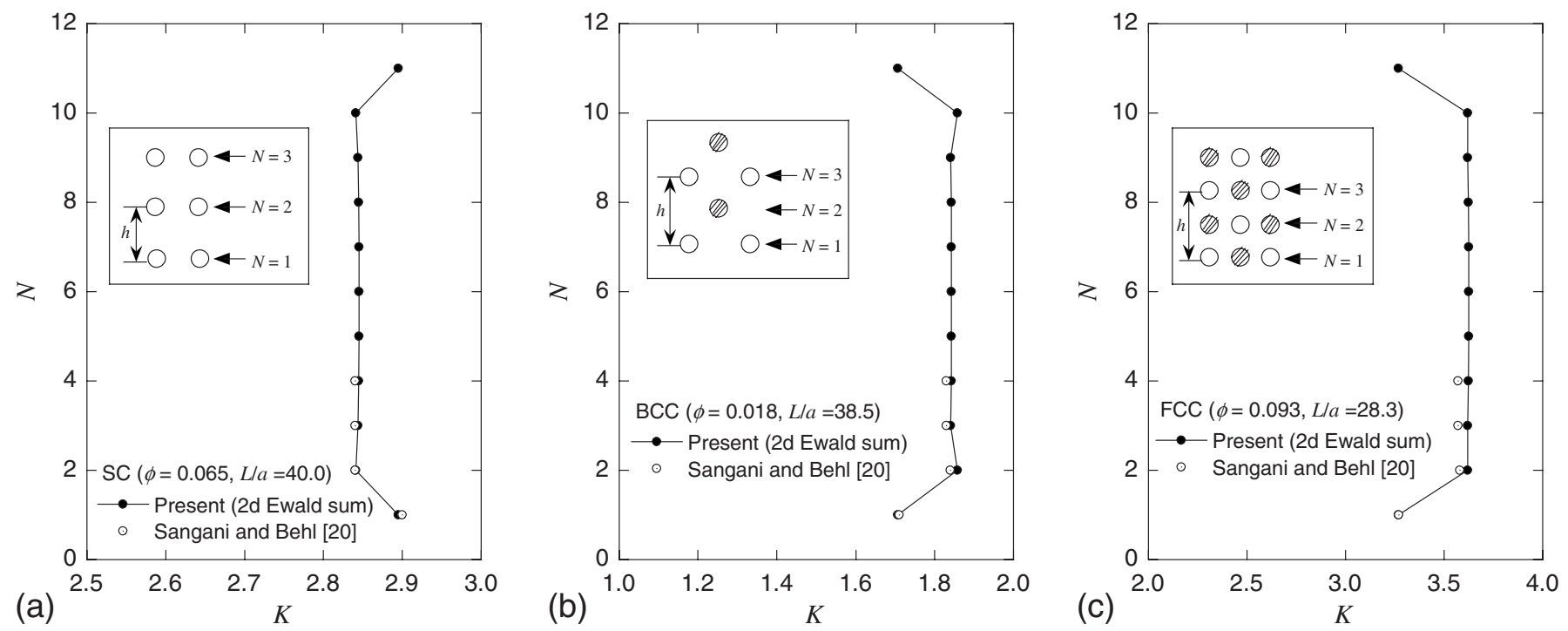

FIG. 3. Drag force acting on a dense particle layer for (a) SC array, (b) BCC array, and (c) FCC array (drag coefficient $K=F / 6 \pi \mu a U$ ).

end of particle layers decays only for a few rows and the drag coefficient is almost constant inside the layer even for the packing limit. It is found from Fig. 3 that our results show excellent agreement with the solutions by Sangani et al. for SC and BCC, while it is slightly different for FCC array because of the larger $\phi$.

Based on the results shown in Figs. 2 and 3, the dynamic behavior of particles in the layer can be inferred as follows. If the arrangement of the particle in the layer is held in some degree, for SC array, the particle near the front end is close to the central particles and the rear particle comes off because the drag force on both particles is larger than that on the centers. On the contrary, the front particle goes away from the central particles and the rear particle is close to them for BCC and FCC arrays. The detailed dynamics of these particle layers are described in the next section.

Figures 4 and 5 show the drag coefficient $K$ at the center of the finite layer in various concentrations. The total number

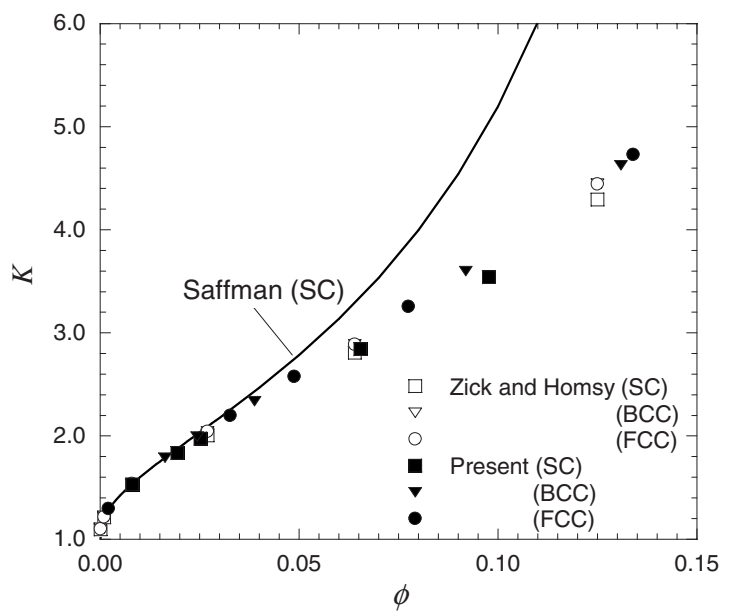

FIG. 4. Drag coefficient $K$ at the center of layer with that in infinite system by Zick and Homsy [18] and analytical solution for dilute regular packing by Saffman [17]. of arrays $N_{L}$ is 15 , which is the same as that shown in Fig. 2 . We adjusted the particle concentration $\phi$ by changing in the unit length of the separation $h / a$. It is found that the drag on the center particle increases with increasing $\phi$. This corresponds to the increase in the permeation resistance of the denser particulate system. Figures also show the numerical results for the infinite packing by Zick and Homsy [18] and the analytical expression by Saffman [17] for the dilute infinite system given by

$$
K=\frac{1}{1-\beta \phi^{1 / 3}},
$$

where $\beta=1.74$ for SC array. The drag coefficient on the center particle in the finite layer is in quantitative agreement with that in the infinite array for $\phi<0.2$. For denser condition, the drag coefficient at the center of the finite layer is

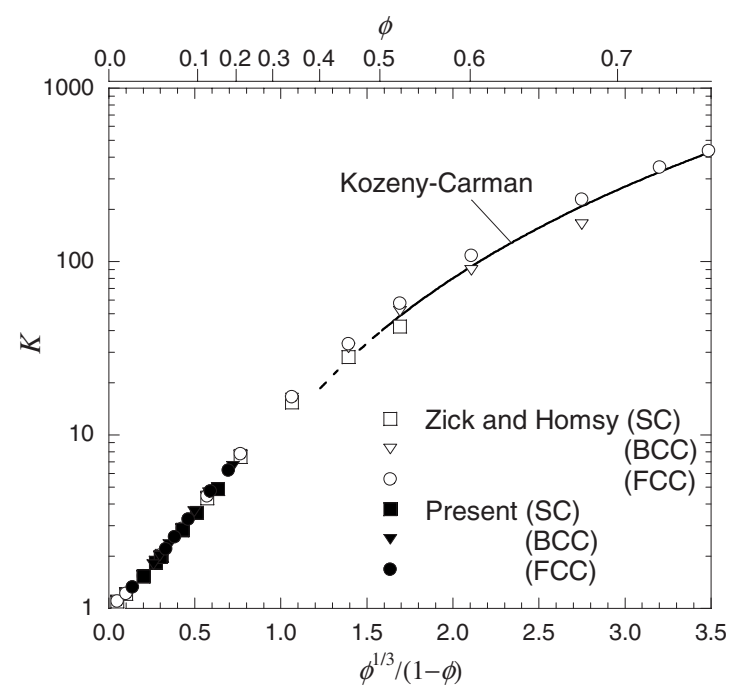

FIG. 5. Drag coefficient $K$ at the center of layer with that in infinite system by Zick and Homsy [18] and drag coefficient calculated by Kozeny-Carman relation $K=10 \phi /(1-\phi)^{3}$. 


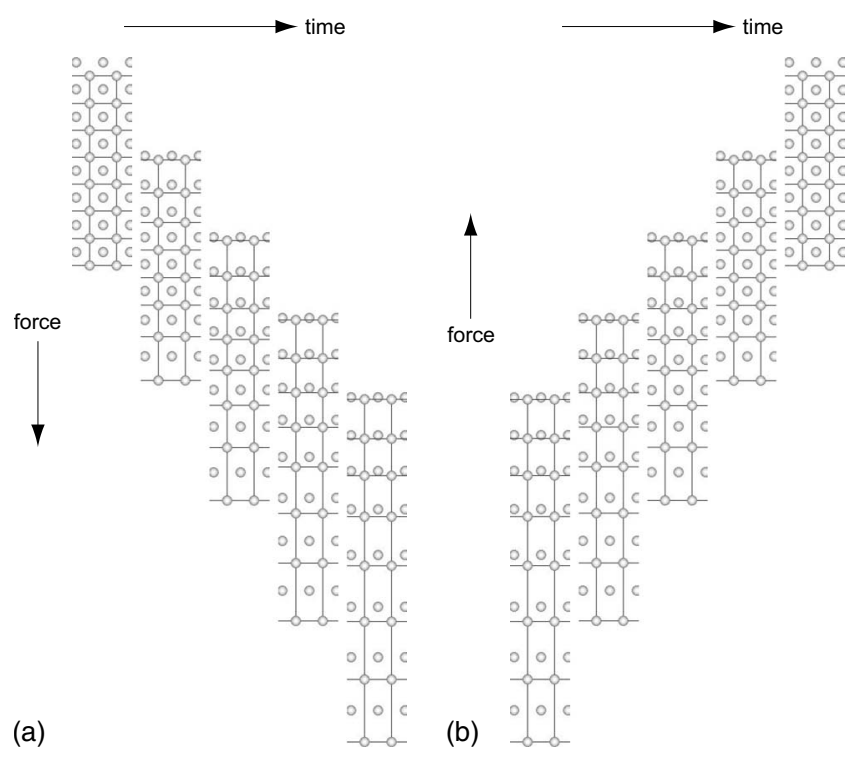

FIG. 6. Relative motion of particle layer to fluid for (a) BCC array $\left(L_{0} / a=40, \phi_{0}=0.055\right)$ and (b) its reverse motion.

close to the drag coefficient of a particle calculated by Kozeny-Carman permeation resistance $K=10 \phi /(1-\phi)^{3}$.

In this section, we have discussed the drag force exerted on particles in a spatially fixed layer. We can summarize findings from the results as follows: (1) the drag on central particles in the layer is almost the same as that in the infinite system; (2) the drag at the end of the layer differs from the center and it depends on particle arrangement inside the layer; (3) the drag difference is confined to the immediate vicinity to the end. On the basis of these results, we take a discussion one step further to the dynamic behavior of particles in the next section.

\section{B. Dynamic behavior of regular particle layer}

In the above section, the drag force exerted on fixed particle layers have been discussed. However, in reality, the relative position of particles may change due to the difference of the drag force on each particle and, consequently, the regularity of the arrangement of particles will be broken. We discuss the dynamic behavior of the regular particle layer, which moves freely in this section.

Figure 6(a) shows the relative motion of BCC layer to fluid for initial thickness $L_{0} / a=40$ and initial concentration $\phi_{0}=0.055$. We obtained these results by solving Eq. (7) with Eq. (8) and then integrating the velocity of all particles numerically. At an early stage, the rear particle approaches to the central particles, while the front particle goes away due to the difference of the drag force. It is consistent with the inference in the above section. However, the end effect is transmitted to the center with time and the relative position of particle becomes complicated. Even though seemingly complex at a glance, the motion of each particle has some rules. Figure 6(b) indicates the results of the reverse motion of BCC layer. To obtain this, we used the final position of particles shown in Fig. 6(a) as an initial condition and applied the external force in the opposite direction. As can be

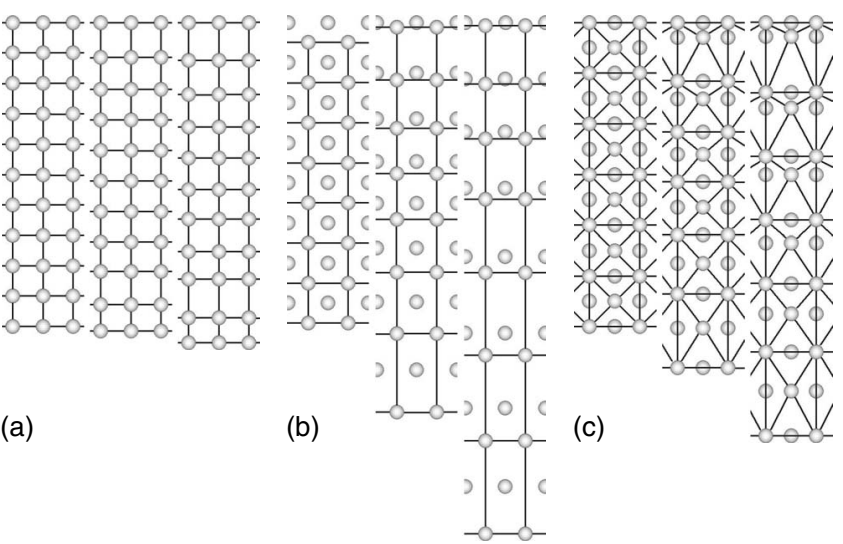

FIG. 7. Relative position of particles in regular arrangement layer for initial thickness $L_{0} / a=40$ and initial concentration (a) $\phi_{0}=0.065$ (SC), (b) $\phi_{0}=0.055$ (BCC), and (c) $\phi_{0}=0.057$ (FCC).

seen, the broken regular arrangement is completely restored to its former state. Such a reversible behavior is due to the kinematic reversibility of the Stokes equation $[21,22]$.

Figure 7 indicates the positions of particles in SC, BCC, and FCC layers observed in the coordinates moving with the rear particle at the same time intervals. The initial layer thickness is $L_{0} / a=40$ and the initial concentration is set to be $\phi_{0} \sim 0.06$ in all layers by adjusting the unit length of separation $h$. As inferred in the above section, the front particle is initially close to the central particles and the rear particle comes off for SC layer and vice versa for BCC and FCC. However, the relative position of particles becomes complicated as time passes. Because of the complicated behavior of particles, it is difficult to describe how the concentration profile changes. Suffice it to say that BCC layer looks dilute at the front and dense at the rear.

It is also found in Fig. 7 that even though the initial thickness and concentration of all layers are similar, the spreading behavior is considerably different from each other. While SC layer does not change its thickness definitely, BCC layer changes the thickness almost 1.5 times as large as the initial one for the same period. These results suggest that the particle arrangement inside the layer greatly affects the overall diffusive motion.

From these results, the motion of the particle layer with the regular arrangement can be explained as follows. If the particle layer has a finite thickness, the drag force exerted on the particle near layer ends is different from the center, and it depends on the particle arrangement inside the layer. If the relative position of particles is fixed, this end effect is restricted to the particle near the ends. However, in case of the dynamic system, the end effect is transmitted into the center of the layer and it greatly changes the inside arrangement. Consequently, the spreading behavior of the layer varies with the inside arrangement even if the initial concentration is similar. These results imply that the hydrodynamic diffusion caused by the end effect depends not only on the concentration but on the inside arrangement of particles. This is a remarkable different point from molecular diffusion, which depends only on the gradient of the concentration.

In actual system, the particles could not keep their regularity as shown in Fig. 7 for a long time, because of the 


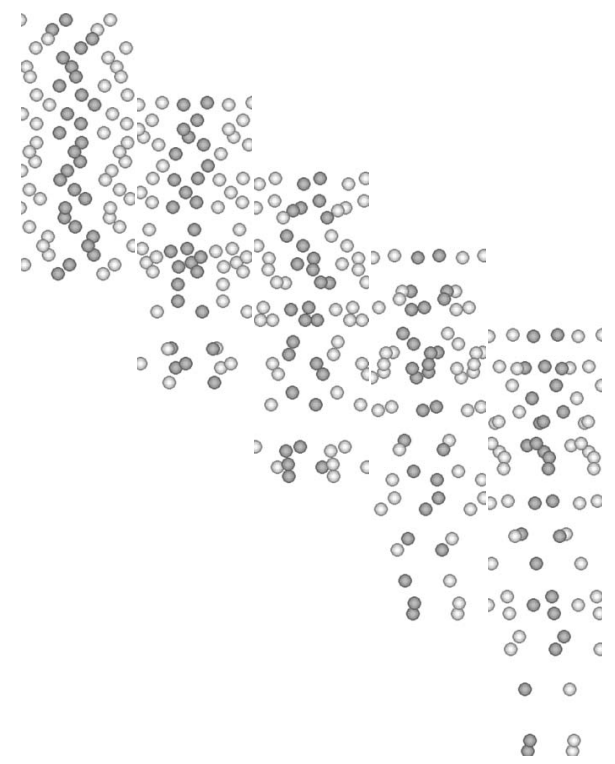

FIG. 8. Relative motion of random particle layer to fluid for initial thickness $L_{0} / a=40$ and $\phi_{0}=0.060$.

clumping instability (the hydrodynamic instability of particles with regular arrangement) $[23,24]$ or the gravityinduced instability (the instability which occurs at suspension-liquid interfaces by gravity) $[25,26]$. Therefore, it must be said that the results shown in this section are somewhat idealistic. They are rather important for discussions of the hydrodynamic effect on random particle layers, which are given in the next section.

\section{Motion of random particle layer}

The relative motion of particle layers with random arrangements to fluid has been also examined. Figure 8 indicates an example of the motion of a random particle layer obtained numerically. In the figure, the behaviors of particles for three lattices of Ewald sum $\left(n_{1}=-1,0,1\right)$ are shown. The initial layer thickness and initial concentration are similar to the results for regular particle layers shown in Fig. 7, and are $L_{0} / a=40$ and $\phi_{0}=0.060$, respectively. It would appear that the profile of the concentration in the layer becomes dilute at the front and dense at the rear, which is similar to BCC layer shown in Fig. 7.

In order to understand the spreading behavior of layers more quantitatively, we calculated the motion of 13 kinds of random particle layers with different arrangements. Figures 9 and 10 indicate the changes in the layer thickness and the concentration of random layers with their movement distance, respectively. These figures also show the results for regular particle layers. As for regular layers, the thicknesses of BCC, FCC, and SC layers become larger in that order. In response to changing the thickness, the overall concentration inside the layer becomes smaller. It is found from Fig. 9 that the random particle layer changes its thickness similarly to BCC layer. Ishii [19] studied the drag difference at the end of semi-infinite particle arrays and reported that its dependency on the inside arrangement is caused by the variance of flow pattern through the layer, which is determined by the posi-

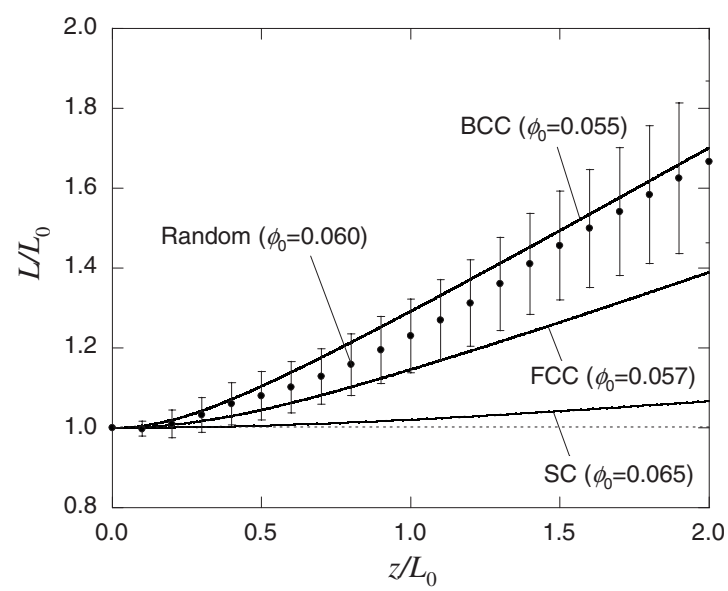

FIG. 9. Layer thickness of random particle layer with those of periodic layer.

tional relation of particles in the direction of movement. The results shown here suggest that the positional relation of particles in random layer and BCC layer are alike hydrodynamically, and, consequently, the random layer diffuses in the similar way to BCC layer.

Figure 11 shows the relationship between the instantaneous concentration and the average velocity of particles in 13 kinds of random particle layers during their relative motion to fluid. The small dots represent our results. It is found that the average velocity of particles in all layers changes in a similar fashion, i.e., the spreading of layers reduces the concentration and, consequently, the average velocity increases in some degree (the plots move from right to left with passing time). For comparison, the velocity of regular infinite array $U / U_{0}=K^{-1} \quad\left(U_{0}=F / 6 \pi \mu a\right.$ : Stokes velocity $)$ given by $\mathrm{Eq}$. (10), and the following theoretical expression for random infinite array on dilute condition [27] are also indicated in the figure:

$$
\frac{U}{U_{0}}=1-6.55 \phi .
$$

If the particles in the layer are perfectly at random and the end effect is minor, it might be expected that the average

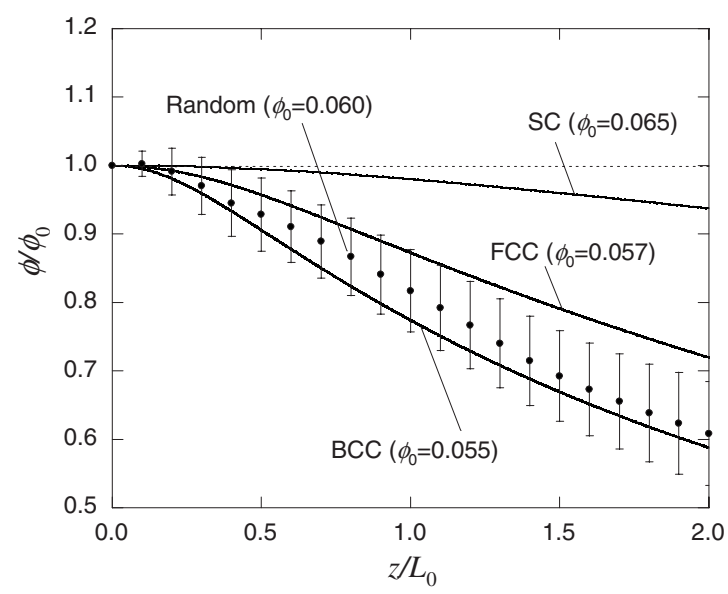

FIG. 10. Particle concentration in random particle layer with those in periodic layer. 


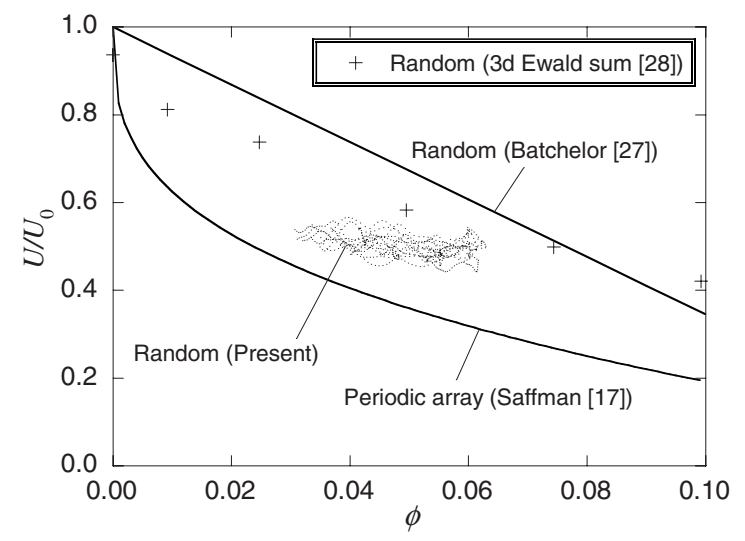

FIG. 11. Average velocity of random particle layer (+: numerical results of infinite system by Phillips et al. [28]).

velocity is similar to Eq. (11), which is the expression for random infinite system. However, our results lie between the results by Eq. (11) and the results of regular array given by Eq. (10). One of the possible reason is that the system treated here is the "periodically random" system, in which random nature of the particle arrangement is not fully realized owing to the long-range hydrodynamic interaction between particles in Stokes flow. Phillips et al. [28] reported the analogous effect in an infinite random system. As shown in Fig.
11 , their calculation results on the velocity of an infinite particle system are close to our results of a finite system. Therefore, it remains possible that our system is not fully random. However, it might be expected that the diffusive behavior of the particle layer, as discussed above is less affected by the periodic randomness, because the positional relation of adjacent particles is a major influence on it.

\section{CONCLUSIONS}

The diffusive behavior of a thin particle layer caused by the hydrodynamic interaction was examined theoretically and numerically. Regarding regularly arranged particle layers, we showed the difference of the drag force between particles near the end and the center of the layer and its dependency on the particle arrangement inside the layer. Owing to such a drag difference, the dynamic behavior of the particle layer also varies with the inside arrangement even if the concentration is similar. Moreover, we discussed the hydrodynamic effect on particle layers with random arrangement. In case of random particle layers, the spreading motion is similar to that of BCC layer. It would appear that the positioning relation of particles in random layer and that in BCC layer are hydrodynamically alike and, consequently, the similar effect is exerted on both layers.
[1] Y. Xia, B. Gates, Y. Yin, and Y. Lu, Adv. Mater. 12, 693 (2000).

[2] R. H. Davis and A. Acrivos, Annu. Rev. Fluid Mech. 17, 91 (1985).

[3] R. H. Davis, J. Fluid Mech. 310, 325 (1996).

[4] J.-Z. Xue, E. Herbolzheimer, M. A. Rutgers, W. B. Russel, and P. M. Chaikin, Phys. Rev. Lett. 69, 1715 (1992).

[5] J. Martin, N. Rakotomalala, and D. Salin, Phys. Fluids 6, 3215 (1994).

[6] J. Martin, N. Rakotomalala, and D. Salin, Phys. Rev. Lett. 74, 1347 (1995).

[7] H. Nicolai and E. Guazzelli, Phys. Fluids 7, 3 (1995).

[8] H. Nicolai, B. Herzhaft, E. J. Hinch, L. Oger, and E. Guazzelli, Phys. Fluids 7, 12 (1995).

[9] L. Bergougnoux, S. Ghicini, E. Guazzelli, and E. J. Hinch, Phys. Fluids 15, 1875 (2003).

[10] S. Kim and S. J. Karrila, Microhydrodynamics (ButterworthHeinemann, Boston, 1991).

[11] J. Rotne and S. Prager, J. Chem. Phys. 50, 4831 (1969).

[12] C. W. J. Beenakker, J. Chem. Phys. 85, 1581 (1986).

[13] K. Ichiki, Master thesis, Tohoku University, 1993.
[14] L. Durlofsky, J. F. Brady, and G. Bossis, J. Fluid Mech. 180, 21 (1987).

[15] D. J. Jeffrey and Y. Onishi, J. Fluid Mech. 139, 261 (1984).

[16] H. Hasimoto, J. Fluid Mech. 5, 317 (1959).

[17] P. G. Saffman, Stud. Appl. Math. 52, 115 (1973).

[18] A. A. Zick and G. M. Homsy, J. Fluid Mech. 115, 13 (1982).

[19] K. Ishii, J. Phys. Soc. Jpn. 46, 675 (1979).

[20] A. S. Sangani and S. Behl, Phys. Fluids 1, 21 (1989).

[21] J. W. Hiby, in Proc. Symp. Interaction between Fluids and Particles (Institute of Chemical Engineers, London, 1962), pp. 312-325.

[22] E. Guyon, J.-P. Hulin, L. Petit, and C. D. Mitescu, Physical Hydrodynamics (Oxford University Press, Oxford, 2001).

[23] J. M. Crowley, J. Fluid Mech. 45, 151 (1971).

[24] S. Ramaswamy, Adv. Phys. 50, 297 (2001).

[25] C. Völtz, M. Schröter, G. Iori, A. Betat, A. Lange, A. Engel, and I. Rehberg, Phys. Rep. 337, 117 (2000).

[26] C. Völtz, Phys. Rev. E 68, 021408 (2003).

[27] G. K. Batchelor, J. Fluid Mech. 52, 245 (1972).

[28] R. J. Phillips, J. F. Brady, and G. Bossis, Phys. Fluids 31, 3462 (1988). 